\title{
The Rostral Medial Prefrontal Cortex Regulates the Expression of Conditioned Eyelid Responses in Behaving Rabbits
}

\author{
Rocío Leal-Campanario, José M. Delgado-García, and Agnès Gruart \\ Division of Neurosciences, Pablo de Olavide University, E-41013 Seville, Spain
}

We studied the contribution of the rostral $\mathrm{mPFC}$ ( $\mathrm{rmPFC}$ ) to the acquisition and performance of classical eyeblink conditioning in rabbits using a delay paradigm. The rmPFC was determined by its afferent projections from the medial half of the mediodorsal thalamic nucleus. The rmPFC neurons were identified by their antidromic activation from the mediodorsal nucleus and/or by their firing characteristics. The rmPFC neurons increased their firing during the first conditioning sessions, but decreased it when conditioned responses (CRs) reached asymptotic values. Therefore, no significant relationships could be established between neuronal firing rates and the percentage of CRs or the electromyographic (EMG) activity of the orbicularis oculi muscle during conditioning. Electrical train stimulation of the rmPFC produced a significant inhibition of air-puff- evoked blinks and reduced the generation of CRs compared with controls. Inhibition of the rmPFC by the local injection of lidocaine produced an increase in the amplitude of evoked reflex and conditioned eyeblinks and in the percentage of CRs. The rmPFC seems to be a potent inhibitor of reflex and conditioned eyeblinks, controlling the release of newly acquired eyelid responses until advanced stages of the acquisition process-i.e., until the need for the acquired response is fully confirmed. Therefore, the rmPFC seems to act as a "flip-flop" mechanism in controlling behavior.

\section{Introduction}

The PFC occupies the highest level in the hierarchical organization of the different cerebral cortex areas and deals with the appropriate selection, timing, and execution of selected behaviors and cognitive processes (Watanabe, 1990; Romo et al., 1999; Fuster, 2001, 2008; Kolb et al., 2004; Nácher et al., 2006; Alexander and Brown, 2011). Although the emotional, behavioral, and cognitive functions performed by the PFC are not easily ascribed to specific prefrontal cortex sites, it is generally accepted that the dorsolateral aspects of the PFC seem to be more related to the sustained neuronal firing underlying working memory processes and to the discriminative components of cognitive phenomena, whereas the orbital and mPFCs are more related to the emotional components of intentional and acquired behaviors, including associative (pavlovian and instrumental) conditioning (Powell et al., 1996; Corbit and Balleine, 2003; Weiss and Disterhoft, 2011; Jurado-Parras et al., 2012).

The involvement of caudal mPFC ( $\mathrm{cmPFC}$ ) areas in different aspects of the acquisition, reversal, and retrieval of classical eye-

\footnotetext{
Received Dec. 4, 2012; revised Jan. 14, 2013; accepted Jan. 21, 2013.

Author contributions: R.L.-C., J.M.D.-G., and A.G. designed research; R.L.-C. performed research; R.L.-C., J.M.D.-G., and A.G. analyzed data; R.L.-C., J.M.D.-G., and A.G. wrote the paper.

This research was supported by the Spanish Ministry of Economy and Competitivity (Grants BFU201129089 and BFU2011-29286) and Junta de Andalucía (Grants BI0122, CVI 2487, and P07-CVI-02686) to A.G. and J.M.D.-G. We thank Mr. Leopoldo Pérez Rosendo for help in animal handling and care and Mr. Roger Churchill for help in manuscript editing.

The authors declare no competing financial interests.

Correspondence should be addressed to Agnès Gruart, Division of Neurosciences, Pablo de Olavide University, Carretera de Utrera, km 1, 41013 Seville, Spain. E-mail: agrumas@upo.es.

DOI:10.1523/JNEUROSCI.5560-12.2013

Copyright $\odot 2013$ the authors $\quad 0270-6474 / 13 / 334378-09 \$ 15.00 / 0$
}

blink conditioning using trace paradigms is well established (Kronforst-Collins and Disterhoft, 1998; Powell et al., 2005). Moreover, the cmPFC seems to be necessary when weak unconditioned stimuli (US) are used and during partial reinforcement and trace conditioning reversal (Powell et al., 1996; KronforstCollins and Disterhoft, 1998; Weible et al., 2003; Simon et al., 2005). In contrast, it has been reported that the rostral mPFC (rmPFC) inhibits the expression of reflex and conditioned eyelid responses potently without affecting the latent acquisition of conditioned responses (CRs; Leal-Campanario et al., 2007). Earlier studies indicated that the electrical stimulation of the orbital PFC inhibits spinal reflexes in cats (Sauerland et al., 1967) and that lesions of the mPFC evoke hyperactivity and resistance to extinction of acquired motor abilities in rabbits (Weible et al., 2000; Kolb et al., 2004). Therefore, the rmPFC could play an adaptive and inhibitory role in the expression of overt motor responses, including reflexively evoked blinks and CRs.

The present experiments were aimed at studying the firing activities of identified rmPFC neurons during classical eyeblink conditioning of behaving rabbits. In addition, we studied the effects of in vivo activation and inactivation of the same rmPFC area during the training process. For conditioning, we used a delay paradigm because it is generally assumed that cerebral cortical areas are not necessary for its proper acquisition and retrieval (Clark et al., 1984; Mauk and Thompson, 1987; Takehara-Nishiuchi et al., 2005; Oswald et al., 2006). In this way, we avoided any disturbing effect of rmPFC activation or inactivation on the acquisition process (Leal-Campanario et al., 2007). The firing activities of contralateral pyramidal rmPFC neurons were recorded across the successive habituation and condi- 

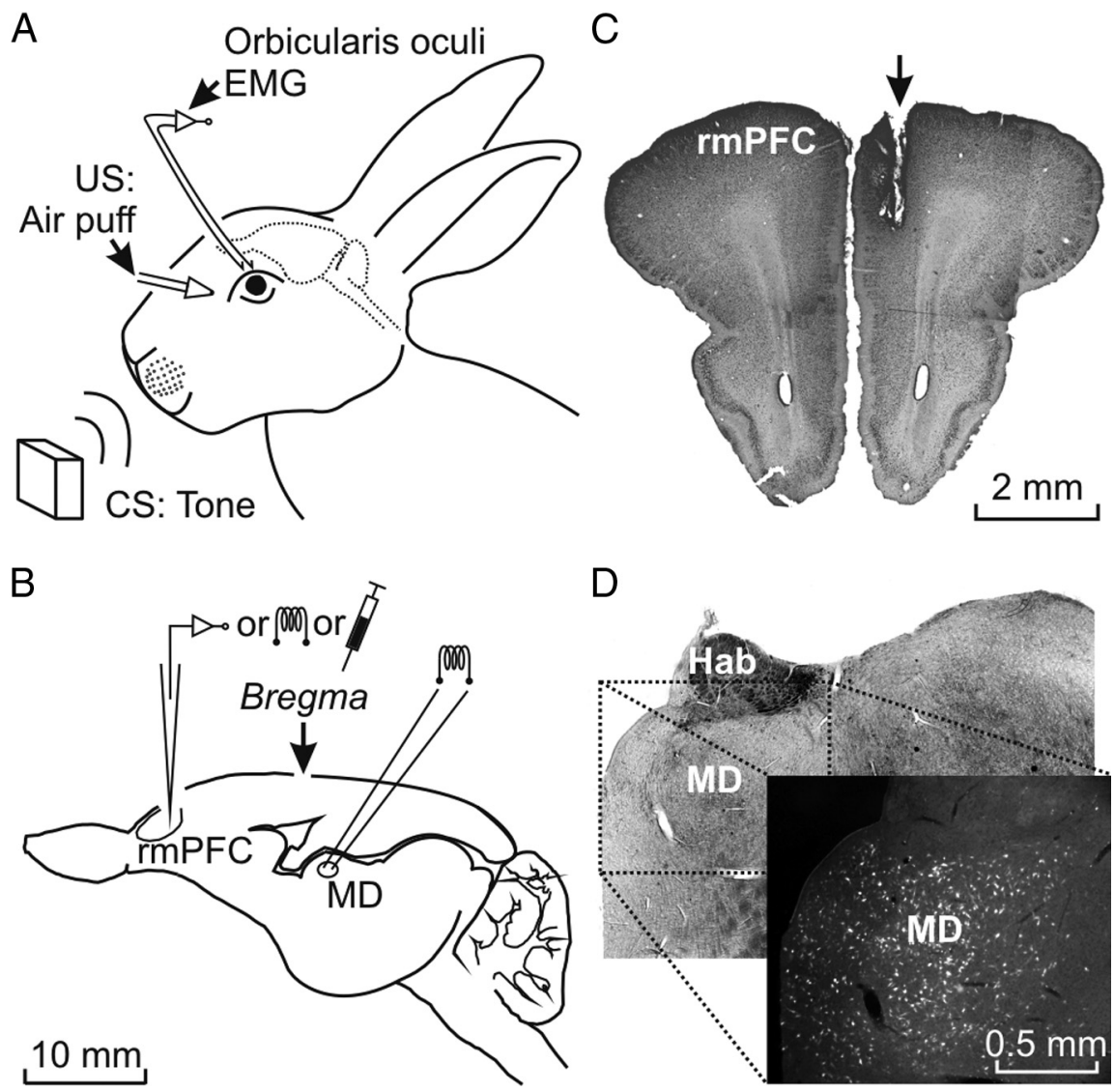

Figure 1. Experimental design. $\boldsymbol{A}$, For the classical conditioning of eyelid responses, animals were chronically implanted with EMG recording electrodes in the left orbicularis oculi muscle. The US consisted of an air puff presented to the ipsilateral cornea and the $C S$ consisted of tones presented biaurally. $\boldsymbol{B}$, Diagram of the rabbit brain illustrating the stimulating, recording, and injection sites. C, Photomicrograph illustrating the location of a stimulated site in the right rmPFC (arrow). D, Photomicrograph illustrating labeled neurons located in the MD nucleus after BDA injection in the rmPFC, as indicated in $\boldsymbol{C}$. Calibrations for $\boldsymbol{B}-\boldsymbol{D}$ are indicated. Hab, Habenula nucleus; MD, mediodorsal thalamic nucleus.

tioning sessions. In addition, the rmPFC was activated (by electrical stimulation) or inactivated (by local lidocaine injections) during selected conditioning sessions. Our results suggest that the rmPFC plays a permissive and/or a releasing/depressing role in the expression of CRs but is not directly involved in the acquisition process or in the proper execution of the acquired eyelid responses.

\section{Materials and Methods}

Experimental animals. Experiments were performed on adult male rabbits (New Zealand white albino) weighing $2.5-3 \mathrm{~kg}$ on arrival, obtained from an authorized supplier (Isoquimen). Animals were housed in individual cages for the whole experiment, and kept on a 12/12 h light/dark cycle with constant ambient temperature $\left(21 \pm 1^{\circ} \mathrm{C}\right)$ and humidity $(50 \pm$ $7 \%)$. Food and water were available ad libitum. All experimental procedures were performed in accordance with the guidelines of the European Union Council (2003/65/CE) and Spanish (BOE 252/34367-91, 2005) regulations for the use of laboratory animals in chronic experiments. Experimental protocols were also approved by the Pablo de Olavide University's local ethics committee.

Surgery. Animals were anesthetized with a ketamine-xylazine mixture (50 mg/ml of Ketaminol, $20 \mathrm{mg} / \mathrm{ml}$ of Rompun, and $0.5 \mathrm{mg} / \mathrm{kg}$ of atropine sulfate). All of the animals were implanted with recording bipolar hook electrodes in the left orbicularis oculi muscle (Fig. 1A). These electrodes were made from Teflon-coated stainless steel wire (A-M Systems) with an external diameter of $50 \mu \mathrm{m}$. In a group $(n=4)$ of animals, a 5 $\mathrm{mm} \times 5 \mathrm{~mm}$ window was drilled in the frontal bone centered above the right rmPFC (Girgis and Shih-Chang, 1981). The dura mater was re- moved and an acrylic recording chamber was constructed around the window. The brain surface was protected with a piece of silicone sheet and the chamber was filled with sterile gauze and capped with a plastic cover. A needle tip was implanted stereotaxically in a chamber corner for reference purposes during unitary recordings. Animals were also implanted with a bipolar stimulating electrode in the ipsilateral mediodorsal thalamic nucleus [anteroposterior $(\mathrm{AP})=-5 \mathrm{~mm}$, lateral $(\mathrm{L})=-1.2 \mathrm{~mm}$, and depth (D) $=-9 \mathrm{~mm}$ from bregma] (Girgis and Shih-Chang, 1981). Finally, a headholding system consisting of three bolts cemented to the skull perpendicular to the stereotaxic plane was implanted (Múnera et al., 2001). A silver electrode (1 $\mathrm{mm}$ in diameter) was attached to the skull as a ground. Terminals of EMG, stimulating, and ground electrodes were soldered to a nine-pin socket. All wire connections were covered with cyanoacrylate glue and the whole system was attached to the skull with the aid of 3 small screws fastened and cemented with an acrylic resin to the bone (for details, see Leal-Campanario et al., 2007).

In addition to being prepared for the EMG recording, a second group $(n=6)$ of animals was additionally implanted with a stimulating electrode in the right $\mathrm{rmPFC}(\mathrm{AP}=9-11 \mathrm{~mm}$, $\mathrm{L}=-1 \mathrm{~mm}$, and $\mathrm{D}=2.5-3 \mathrm{~mm}$ ) (Girgis and Shih-Chang, 1981; Shek et al., 1986). In another group $(n=6)$ of animals, a $4 \times 4 \mathrm{~mm}$ window was drilled in the frontal bone centered on the rmPFC (Girgis and Shih-Chang, 1981; Shek et al., 1986) and a 22-gauge guide cannula (C313G; Plastic One) was implanted at the above-indicated (AP and L) coordinates but at a depth of $2 \mathrm{~mm}$ from bregma. The dura mater surface was protected with a silicone rubber cover (SILASTIC; Dow Corning) and the window closed with acrylic resin.

Additional control, stimulated, or lidocaine-injected animals were used for recording reflexively evoked eyeblinks and for the proper immunohistological identification of the rmPFC site (see below).

Recording and stimulation procedures. Recording sessions began 2 weeks after surgery. Each rabbit was placed in a Perspex restrainer specially designed for limiting the animal's movements (Gruart et al., 2000). The box was placed on the recording table and was surrounded by a black cloth. The recording room was kept softly illuminated and a $60 \mathrm{~dB}$ background white noise was switched on during the experiments. For all subjects, the first 2 recording sessions consisted of adapting the rabbit to the restrainer and to the experimental conditions; no stimulus was presented. As indicated above, animals were divided into 4 experimental groups: controls, animals for electrical stimulation of the rmPFC, animals for local lidocaine administration in the rmPFC, and animals for unitary recordings of rmPFC neurons.

The EMG activity of the selected muscle was recorded with differential amplifiers with a bandwidth of $0.1 \mathrm{~Hz}$ to $10 \mathrm{kHz}$ (P511; GrassTelefactor). Neuronal electrical activity was recorded in the rmPFC area with the help of a NEX-1 preamplifier (Biomedical Engineering). These recordings were performed with glass micropipettes filled with $2 \mathrm{M} \mathrm{NaCl}$ (3-6 M $\Omega$ of resistance) and filtered in a bandwidth of $1-10 \mathrm{kHz}$. Field potentials were recorded with low-resistance electrodes (1-3 M $\Omega$ ) or were low-pass filtered $(>100 \mathrm{~Hz})$ from unitary recordings. The recording area was approached with the help of stereotaxic coordinates (Girgis and Shih-Chang, 1981; Shek et al., 1986), and antidromic and orthodromic (i.e., synaptic) field potentials were evoked by electrical stimulation of the mediodorsal thalamic nucleus. Using this electrophysiological 
approach, we avoided mixing the behavior of different neuronal types presenting different input-output organizations. Criteria to determine whether the recorded and the activated neuron were the same and to discriminate somatic versus axonic recordings were followed systematically (Múnera et al., 2001 for details). At the end of each recording session, the recording micropipette was always removed and the recording chamber sterilized and closed.

Air puffs directed at the left cornea were applied through the opening of a plastic pipette ( $3 \mathrm{~mm}$ in diameter) attached to a metal holder fixed to the animal's nine-pin socket (dual-channel air-puff device; Biomedical Engineering). Tones were applied from a loudspeaker located $80 \mathrm{~cm}$ below the animal's head. Electrical stimulation of the rmPFC and of the mediodorsal nucleus was achieved by an isolation unit. Single (cathodal, square, $50 \mu \mathrm{s},<500 \mu \mathrm{A}$ pulses) and train $(200 \mathrm{~Hz}, 50 \mathrm{~ms})$ stimuli were programmed with a CS-220 stimulator across an isolation unit (ISU-220; Cibertec).

The electrical stimulation applied to the contralateral rmPFC during reflexively evoked eyeblinks and during the classical conditioning of eyelid responses was applied $80 \mathrm{~ms}$ before air-puff presentation, and consisted of a train of electrical pulses of $200 \mathrm{~Hz}, 50 \mathrm{~ms}$ in duration, and $<500 \mu \mathrm{A}$ of intensity ending $30 \mathrm{~ms}$ before the US onset. It has been reported previously that the maximum inhibitory effects with the minimum stimulus intensity $(\mathrm{mA})$ were obtained when the train was presented $80 \mathrm{~ms}$ before air-puff presentation (Leal-Campanario et al., 2007).

Classical conditioning. As described in detail previously (LealCampanario et al., 2007), classical eyeblink conditioning was achieved by the use of a delay conditioning paradigm. For this, animals were presented with a tone $(350 \mathrm{~ms}, 600 \mathrm{~Hz}$, and $90 \mathrm{~dB})$ as a conditioned stimulus (CS), followed $250 \mathrm{~ms}$ from its beginning by an air puff (100 $\mathrm{ms}, 3 \mathrm{~kg} / \mathrm{cm}^{2}$ ) as a US. The conditioning session consisted of 66 CS-US trials separated at random by intervals of $50-70 \mathrm{~s}$. Six of the 66 trials were test trials in which the CS was presented alone. A conditioning session lasted for $\sim 80 \mathrm{~min}$ and animals were trained on 10 successive days. An animal was considered conditioned when it was able to produce $80 \%$ of CRs per session to the CS-US paired presentation. For habituation and extinction, animals were presented with the CS alone for the same number of trials/session and at the same time intervals. Animals received two habituation and five extinction sessions. Pseudoconditioning sessions also consisted of 66 trials separated at random by intervals of 50-70 s. For each trial, the CS was presented unpaired in relation to the US, the only restriction being that no more than two CS or US trials occurred sequentially (Gruart et al., 2000). The total training per session for pseudoconditioning was the same as for conditioning.

For the experiments illustrated in Figures 2 to 4 , animals were recorded in the rmPFC area during habituation, conditioning, and extinction sessions. Neuron isolation and identification procedures were performed during the time intervals in which CS-US pairs of stimuli were not presented. Animals included in the rmPFC-stimulated group (see Figs. 5, 6) received a train $(200 \mathrm{~Hz}, 50 \mathrm{~ms})$ of electrical stimuli in the selected rmPFC site. The train started $80 \mathrm{~ms}$ before US presentation. Finally, animals included in the lidocaine group were locally injected with a $5 \%$ lidocaine solution (Brain Medical) at a rate of $0.1 \mu \mathrm{l} / \mathrm{min}$ with the help of a microinfusion pump (model 310; KD Scientific) throughout the conditioning session.
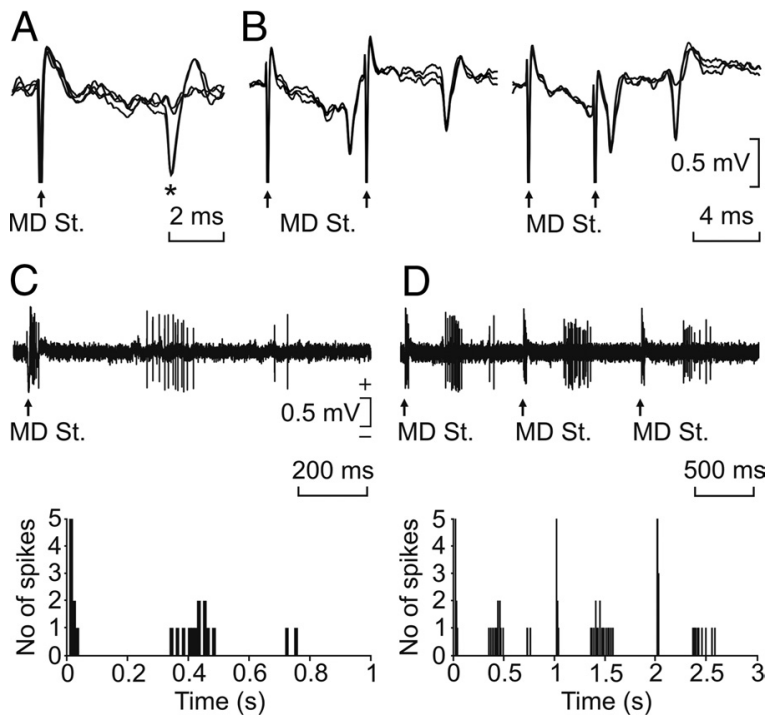

Figure 2. Neuronal identification procedures. $\boldsymbol{A}$, All-or-none antidromic activation of a rmPFC neuron by the stimulation of the ipsilateral mediodorsal thalamic nucleus (MDSt.). $\boldsymbol{B}$, Collision test for an rmPFC neuron activated from the MD nucleus. $\boldsymbol{C}, \boldsymbol{D}$, Synaptic activation of an identified rmPFC neuron represented with two different time bases. The stimulus consisted in a pair of pulses (100 $\mu \mathrm{s}$ in duration, $1 \mathrm{~ms}$ of interpulse interval, and up to $2 \mathrm{~mA}$ ) presented at a rate of $1 \mathrm{~Hz}$. The cumulative records for the synaptic activation of this neuron are represented in the two bottom graphs.
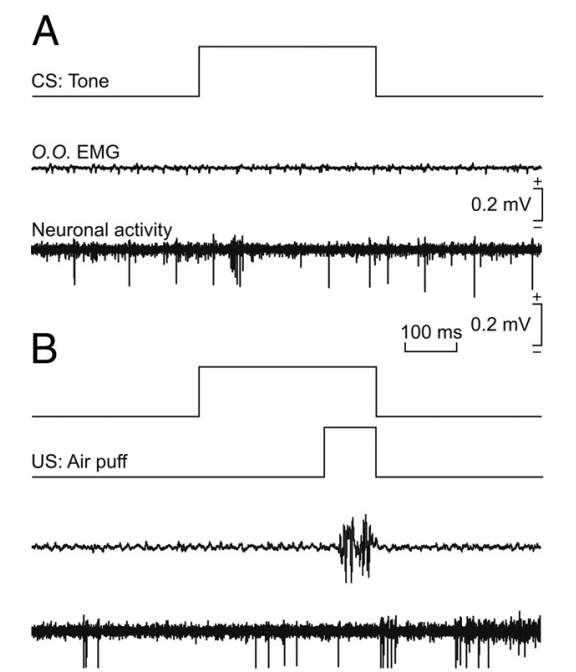

C
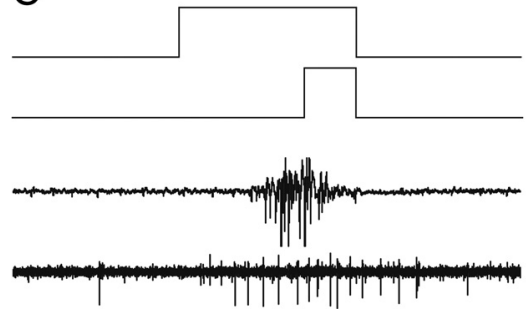
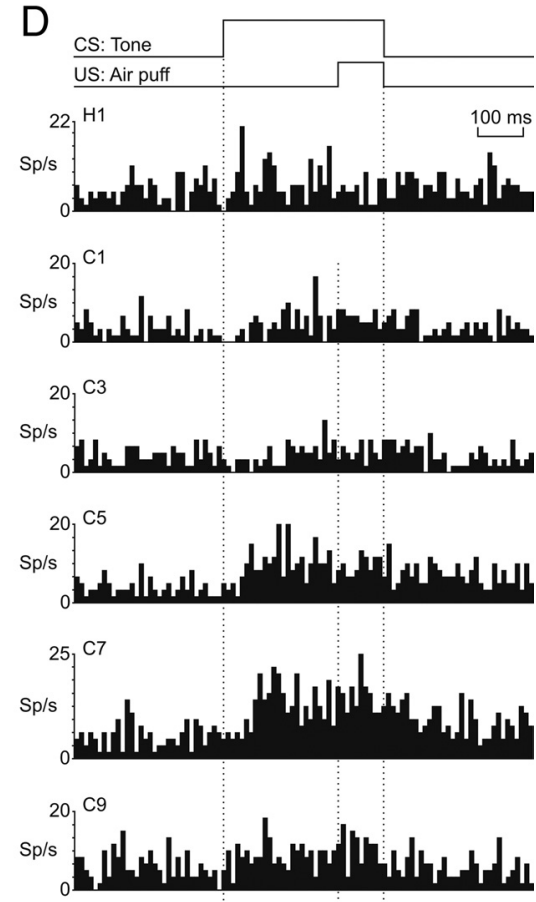

Figure 3. Firing rate of $\mathrm{rmPFC}$ neurons during classical conditioning of eyelid responses. $A$, Representative examples of neuronal activity of rmPFC neurons and the electromyographic activity of the orbicularis oculi muscle $(0.0$. EMG). From top to bottom are illustrated CS presentation, the 0.0. EMG, and the activity of an rmPFC neuron. Records were collected during the first habituation session. $\boldsymbol{B}, \boldsymbol{C}$, Similar sets of records (including air puff, US, presentation) collected during the second $(\boldsymbol{B})$ and the fifth $(\boldsymbol{C})$ conditioning sessions. Calibrations for $\boldsymbol{A}-\boldsymbol{C}$ are indicated. $\boldsymbol{D}$, Average of the firing rates (in spikes $/ \mathrm{s} ; \mathrm{sp} / \mathrm{s}$ ) of $\mathrm{rmPFC}$ neurons recorded during the habituation $(\mathrm{H} 1)$, conditioning $(\mathrm{C} 1, \mathrm{C}, \mathrm{C}, \mathrm{C}, \mathrm{C})$, and extinction (E1) sessions. The delay conditioning paradigm is indicated at the top. The dotted lines indicate the start and end of CS and US presentations. Note that a noticeable increase of neural firing in relation to the paired CS-US presentation took place only around the fifth-seventh sessions. 


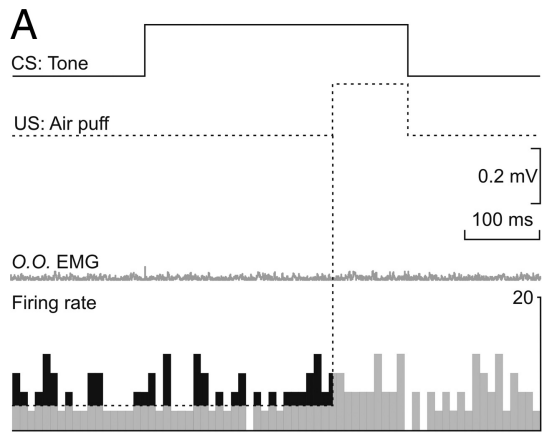

C

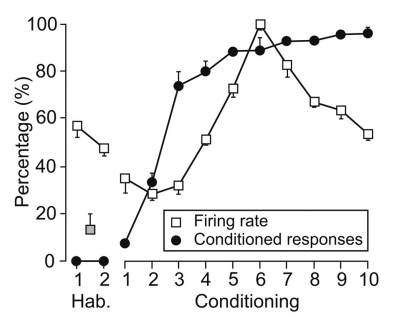

D

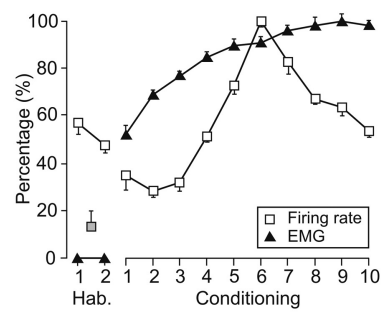

E

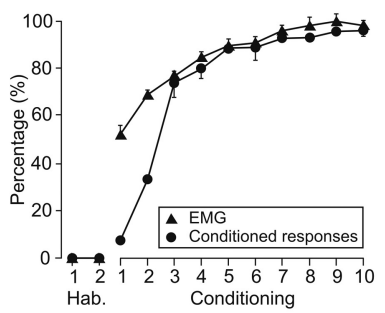

F

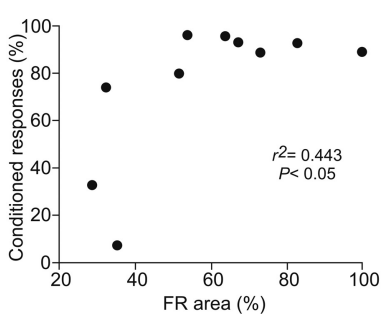

G

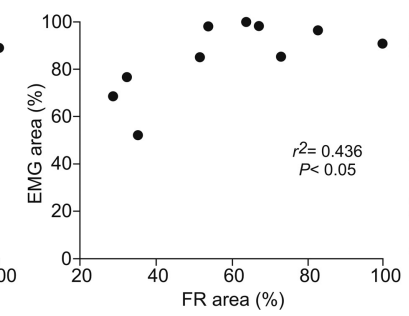

\section{$\mathrm{H}$}

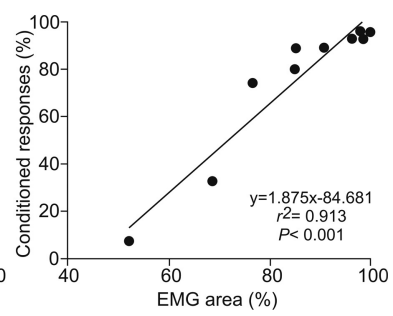

Figure 4. Comparative analysis of the learning curve and the evolution of the electromyographic activity of the orbicularis oculi muscle (0.0. EMG) and of the firing rate of rmPFC neurons across conditioning sessions. $\boldsymbol{A}, \boldsymbol{B}$, Experimental design. From top to bottom are illustrated CS and US presentations, the EMG activity of the 0.0. muscle, and the firing rate of an rmPFC neuron. The illustrated example in $\boldsymbol{A}$ was collected from the habituation session 1; the example in $\boldsymbol{B}$ was collected from the sixth session of a representative animal. Calibrations are as indicated. The respective increases (\%) in the EMG activity of the 0.0. muscle (area, in $\mathrm{mV} \times \mathrm{s}$ ) and in the neuron firing rate (area, in arbitrary units: firing rate $\times \mathrm{s}$ ) taking place during the $(S$-US interval were collected for quantitative analysis across the successive habituation and conditioning sessions. $\boldsymbol{C}-\boldsymbol{E}$, Results of the quantitative analysis illustrated in $\boldsymbol{A}$ and $\boldsymbol{B}$. For comparative purposes, the learning curve obtained by the application of the selected criterion is also illustrated (black circles, in percentage of (Rs, left $y$-axis in $\boldsymbol{C}$ and $\boldsymbol{E}$ ). In addition, the evolution of 0.0 . EMG (black triangles, in percentage of change, left $y$-axis in $\boldsymbol{D}$ and $\boldsymbol{E}$ ) and neuronal firing rate areas (white squares, in percentage of change, left $y$-axis in $\boldsymbol{C}$ and $\boldsymbol{D}$ ) are also illustrated. Note that the percentage of CRs and the area of the evoked EMG responses run in parallel, whereas neuronal firing rate areas peaked in the sixth session but decreased rapidly afterward. $\boldsymbol{F}-\boldsymbol{H}$, Quantitative analysis of the relationships between firing rate area and the percentage of $(R s(\boldsymbol{F})$, firing rate area and EMG area $(\boldsymbol{G})$, and EMG area and the percentage of $\mathrm{CRs}(\boldsymbol{H})$. Each point represents the mean value collected from a single animal during the corresponding session. Only data collected from conditioning sessions are illustrated. Regression lines and their corresponding equations are included only for coefficients of determination $\left(r^{2}>0.6\right)$. The $p$ values for each regression analysis are indicated.

Histology. At the end of the experiments, animals were deeply anesthetized with sodium pentobarbital $(50 \mathrm{mg} / \mathrm{kg}$, i.p.) and perfused transcardially with saline and $4 \%$ paraformaldehyde. The proper location of eyelid EMG electrodes was checked. To determine the final location of rmPFC-stimulating electrodes and injection cannulas, the brain was removed and cut into slices $(50 \mu \mathrm{m})$ and the relevant cortical areas were processed for Nissl staining (Fig. 1C).

Biotinylated dextran amine (BDA; molecular weight 10,000; Invitrogen) was used to identify subdivisions of the mPFC on the basis of its thalamic input (Benjamin et al., 1978; Leal-Campanario et al., 2007). Multiple ( $n=3-5)$ BDA pressure injections were performed in 4 animals using a Picospritzer II device (Parker Hannifin). Injections were made with a glass micropipette ( $20 \mu \mathrm{m}$ tip diameter, $0.5 \mu$ l per injection) in the same prefrontal site selected for microstimulation (AP $=9-11 \mathrm{~mm}, \mathrm{~L}=$ $1 \mathrm{~mm}, \mathrm{D}=2.5-3 \mathrm{~mm}$ from bregma; Girgis and Shih-Chang, 1981; Shek et al., 1986) (Fig. 1C). After 2 weeks of survival time, animals were intracardially perfused with $4 \%$ paraformaldehyde in $\mathrm{PB}, 0.12 \mathrm{M}$. Frozen 50 $\mu \mathrm{m}$ sections were rinsed in PBS, $3 \times 10 \mathrm{~min}$, blocked in 3\% normal horse serum, $4 \%$ bovine serum albumin, and $0.1 \%$ Triton X-100 in PBS for $1 \mathrm{~h}$, and incubated in streptavidin-Cy3 (1: 200; Jackson ImmunoResearch) for $1.5 \mathrm{~h}$. After rinsing in PBS, sections were coverslipped with Citifluor. Retrograde-labeled cells in the mediodorsal thalamic nucleus were photographed using a Leica DM5000 B microscope (Reiner et al., 2000).

Data collection and analysis. The unrectified EMG activity of the recorded muscles, the unitary activity recorded in the $\mathrm{mPPF}$, and $1 \mathrm{~V}$ rectangular pulses corresponding to $\mathrm{CS}$, US, and rmPFC stimuli presented during the different experimental sessions were acquired online through an 8-channel analogto-digital converter (model 1401-plus; CED), and transferred to a computer for quantitative offline analysis. Data were sampled at $5000 \mathrm{~Hz}$ (for EMG recordings) or 25,000 Hz (for unitary recordings) with an amplitude resolution of 12 bits. Computer programs (Spike2 and SIGAVG from CED) were used to display EMG and unitary activities. These programs enabled the quantification, with the aid of cursors, of the onset latency, duration, peak amplitude, and area of the rectified EMG activity of the orbicularis oculi muscle and the latency of unitary recordings. When necessary for quantitative analysis, segments containing CRs were selected exclusively from those obtained during the presentation of the CS alone. The programs also allowed the representation of the firing rate of the recorded neurons (Múnera et al., 2001; Leal-Campanario et al., 2007).

Statistical analyses were performed using the SPSS statistical software package (SPSS) for a statistical significance level of $p=0.05$. Unless otherwise indicated, mean values were calculated from $\geq 20$ measurements collected from three animals. Mean values are followed when necessary by SEM. Collected data were analyzed using a one-way or two-way ANOVA test with time or session as repeated measures, which was coupled with contrast analysis when appropriate. Repeated-measures ANOVA allowed checking the statistical differences of the same group across sessions. The StudentFisher $t$ test or the Shapiro-Wilk H coefficients were used when necessary.

\section{Results}

Identification of the rmPFC recording, stimulation, and injection sites

As illustrated in Figure $1 A$, animals were prepared for chronic recording of the EMG activity of the orbicularis oculi muscle. In addition, animals were prepared for unitary recordings in the rmPFC, the chronic stimulation of this cortical area, or the local administration of lidocaine (Fig. 1B). Stimulating electrodes were implanted in the mediodorsal thalamic nucleus for the antidromic and/or synaptic identification of neurons recorded in the rmPFC (Fig. 1B). As illustrated in Figure $1 C$, the histological examination of the recording, stimulation, or injection sites in- 
dicated that the studied area was restricted to the $\mathrm{rmPFC}(\mathrm{AP}=9-12 \mathrm{~mm}, \mathrm{~L}=-1$ $\mathrm{mm}$, and $\mathrm{D}=2.1-3.8 \mathrm{~mm}$ from bregma; Girgis and Shih-Chang, 1981; Shek et al., 1986), corresponding to the rostral aspect of Brodmann's area 24 or anterior cingulate cortex, and to the rostrodorsal part of Brodmann's area 32 or prelimbic cortex. Stimulating electrodes implanted in the mediodorsal thalamic nucleus were also restricted to this thalamic area $(\mathrm{AP}=-5$ $\mathrm{mm}, \mathrm{L}=-1.2 \mathrm{~mm}$, and $\mathrm{D}=9 \mathrm{~mm}$ from bregma; not illustrated).

As a further proof that those recording and stimulation sites corresponded to the rmPFC and to the mediodorsal thalamic nucleus, we injected BDA (used here as a retrograde tracer) in the $\mathrm{rmPFC}$ areas included in this study. It has already been reported (Benjamin et al., 1978; LealCampanario et al., 2007) that the medial half of the mediodorsal thalamic nucleus projects preferentially to the rmPFC. Figure $1 D$ shows that BDA injections performed in 4 animals selectively labeled the medial part of the mediodorsal thalamic nucleus $(>150$ cell counts $/ \mathrm{mm}^{2}$ ), with a more diffuse labeling of its lateral area. These microinjections seemed not to diffuse to the premotor and/or motor cortices, because labeling of thalamic ventral anterior and ventral lateral nuclei was rather sparse $(<5$ cell counts/ $\mathrm{mm}^{2}$ ).

Across the whole experiment, a total of 189 neurons were recorded in the rmPFC area. As shown in Figure 2, $A$ and $B$, neurons were identified by their antidromic activation from the mediodorsal thalamic nucleus. Only those with an antidromic latency $\leq 6 \mathrm{~ms}$ and a spike duration $>0.5 \mathrm{~ms}$ were considered to be pyramidal neurons $(n=47)$. The spontaneous firing rate of recorded cells ranged from 2 to 12 spikes/s. Characteristically, rmPFC pyramidal cells fired in response to paired pulses $(100 \mu \mathrm{s}$ in duration, $1 \mathrm{~ms}$ of interpulse interval, and up to $2 \mathrm{~mA}$ ) presented to the mediodorsal thalamic nuclei (Fig. $2 C, D$ ). Thalamic activation of rmPFC cells took place as an early $(\sim 10 \mathrm{~ms})$, strong ( $>100$ spikes/s) burst of activity, followed by a delayed $(>350$ $\mathrm{ms})$, weaker $(<50 \mathrm{spikes/s})$ firing (Fig. $2 C, D)$. Interestingly, these delayed bursts of activity were similar in duration and profile to those presented by the pyramidal neurons across conditioning sessions (see below). Additional, non-antidromically identified units $(n=65)$ recorded in the same $\mathrm{rmPFC}$ area but presenting similar spike duration, spontaneous firing rates, and synaptic activation from the mediodorsal thalamic nucleus were also included in this study.

With regard to their firing properties, most neurons recorded and analyzed appeared to be similar to the regular-spiking, slowadapting pyramidal cells described by Dégenètais et al. (2002); that is, they were capable of generating a tonic firing during the CS-US interval when CRs were generated (see below).

\section{Firing properties of rmPFC neurons during classical conditioning of eyelid responses}

Figure $3 A-C$ shows representative examples of rmPFC neurons recorded during the classical eyeblink conditioning study. Figure $3 A$ illustrates the spontaneous firing of an antidromically identified rmPFC pyramidal cell recorded during the first habituation session. In this experimental situation, there was no EMG activity in response to CS (tone) presentation and the recorded neuron presented a low (12 \pm 2 spikes/s) and irregular firing rate. Figure $3 B$ illustrates the firing activity of another rmPFC pyramidal cell recorded during the second conditioning session. The experimental animal did not yet perform a CR (Fig. 3B, EMG trace), but the recorded neuron presented some characteristic short-lasting bursts of activity, mainly at the end of the paired CS-US presentation. Finally, Figure $3 C$ illustrates the firing activity of an rmPFC pyramidal cell during the CS-US interval. This set of recordings was collected during the fifth conditioning session. At this stage, a CR was evident in the EMG trace.

Figure $3 D$ shows the averaged mean firing rates of rmPFC neurons recorded across the successive habituation, conditioning, and extinction sessions collected from a representative animal. A range of $2-5$ identified pyramidal neurons $(n=189)$ were recorded per session $(n=14)$ and animal $(n=3)$. As already indicated, the mean spontaneous firing rate of the recorded neurons was quite low (1-12 spikes/s). Interestingly, this spontaneous firing rate started to increase at the end of the paired CS-US presentations (Fig. 3D, conditioning sessions C1 and C3). The increase in neuronal firing rate taking place during the CS-US interval decreased in latency with respect to the beginning of the CS with successive conditioning sessions (Fig. 3D, conditioning sessions C5 and C7) and also increased in firing frequency. Finally, this CS-US-evoked change in firing started to disappear with successive conditioning sessions once the animal had acquired the associative learning task (Fig. 3D, conditioning session C9) and it was unnoticeable (i.e., it had returned to baseline values) during the first extinction session (Fig. 3D, E1).

Figure 4 shows a comparative analysis of the evolution of the percentage of CRs (determined following the selected criterion), the evolution of the EMG area (in $\mathrm{mV} \times \mathrm{s}$ ) corresponding to the CR (Fig. $4 A, B$, middle trace, black-marked area), and the changes in firing rate related to CRs across conditioning (spikes/s; Fig. $4 A, B$, bottom trace, black-marked area). The aim was to determine whether there was any relationship between learning and the firing rate of rmPFC neurons. As shown in Fig- 

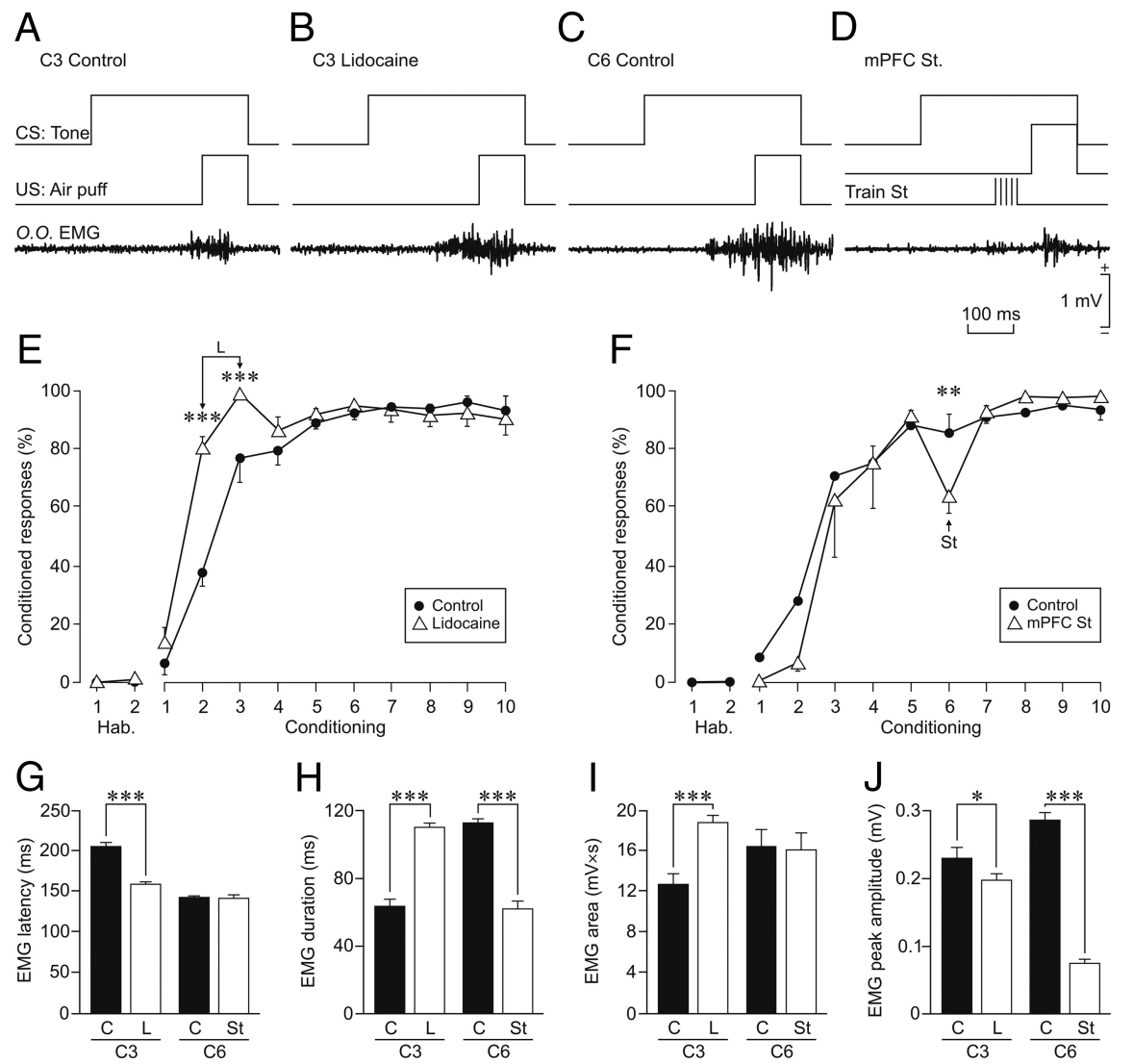

Figure 6. Effects on conditioned eyelid responses of train stimulation of, or local lidocaine administration into, the rmPFC. $A$, Representative recordings of the EMG activity of the orbicularis oculi muscle (0.0. EMG) evoked by a paired CS-US presentation. The EMG trace is the average of 5 single recordings. Records were collected from the third conditioning session from a representative control animal (C3 Control). B, Averaged recording collected from the third conditioning session from an animal with a local lidocaine infusion into the rmPFC performed during the third conditioning session (C3 Lidocaine). C, Averaged recording collected from the sixth conditioning session (C6 Control) of a representative control animal. D, Averaged recording collected from a representative animal stimulated in the rmPFC at the indicated time (St) during the sixth conditioning session (rmPFC St). $\boldsymbol{E}$, Effects of local lidocaine infusion into the rmPFC on the learning curve for the two injection days ( $(2$ and $\mathrm{C} 3$ ). $\boldsymbol{F}$, Effects of rmPFC stimulation on the learning curve (C6). G-J, Quantitative analysis of EMG collected data for the three experimental situations: control (C, black bars), lidocaine administration into the $\operatorname{rmPFC}(L$, white bars), and rmPFC stimulation (St, white bars). The following parameters were quantified: EMG latency ( $\boldsymbol{G}$, in ms for the start of EMG activity corresponding to the conditioned eyelid response), EMG duration $(\boldsymbol{H}$, in ms for EMG activity for the complete conditioned response), integrated EMG area (I, in $\mathrm{mV} \times \mathrm{s}$ ), and EMG peak amplitude $(J$, in $\mathrm{mV})$. For lidocaine, comparison was made with data collected in control and injected animals during the third conditioning session (C3). For train stimulation, comparison was made with data collected during the sixth conditioning session (C6). Significant differences are indicated: ${ }^{*} p<0.05$; ${ }^{* *} p<0.01$; ${ }^{* * *} p<0.001$, two-way ANOVA.

ure $4 C$, the increase in the percentage of CRs (black circles) was paralleled by a similar increase in the mean firing rate presented by rmPFC neurons (white squares) for the CS-US interval across the conditioning sessions 1-6. Interestingly, from conditioning sessions $7-10$, the mean firing rate decreased while the percentage of CRs reached the maximum level. Figure $4 D$ shows an increase in the total area of the EMG activity of the orbicularis oculi muscle corresponding to the acquired CRs (black triangles) and the mean firing rate presented by rmPFC neurons (white squares) for the CS-US interval across conditioning sessions 1-6. From conditioning sessions $7-10$, the mean firing rate decreased and the total area of the EMG reached an asymptotic value. Finally, Figure $4 E$ shows the paralleled increase across conditioning sessions 1-10 in the percentage of CRs (black circles) and in the total area of the EMG activity of the orbicularis oculi muscle corresponding to the acquired CRs (black triangles). In accordance with these results, the changes in firing rate observed in rmPFC neurons during the classical conditioning of eyelid responses using a delay para-

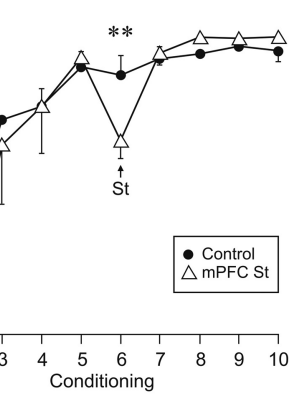

digm seem not to be related to the acquisition process (i.e., to the learning curve) or to the execution of the acquired CR (i.e., to the EMG area). The pseudoconditioned group failed to reach criterion ( $>80 \%$ of CRs) by the 10 th conditioning session (data not shown), so they were excluded from further analysis.

As shown in Figure $4 F-H$, relationships between the rectified EMG area and the percentage of CRs fitted a linear regression model having a high coefficient of determination $\left(r^{2}=0.913, p<0.001\right.$; Fig. $4 H$ ). In contrast, the quantified firing rate area presented lower coefficients of correlation when plotted against the percentage of CRs $\left(r^{2}=0.443, p<0.05\right.$; Fig. $4 F)$ or against the rectified EMG area $\left(r^{2}=\right.$ $0.436, p<0.05$; Fig. $4 G$ ). In fact, these two relationships gave a polynomial fit $\left(r^{2}>0.5\right.$, $p<0.045)$ with an inverted-U shape, suggesting no direct causal relationships between the involved variables.

Generally, these results are consistent with the decrease in mean firing rate of rmPFC neurons shown in Figure 3D session 9 with respect to session 7 , which further proves that these neuronal changes are not learning specific.

\section{Effects of rmPFC activation and} inactivation on reflexively evoked and classically conditioned eyelid responses To determine the precise contribution of the $\mathrm{rmPFC}$ region to the genesis and maintenance of conditioned eyelid responses, we designed a second series of experiments in which we activated the rmPFC by electrical stimulation or inactivated it by the local injection of lidocaine (a local anesthetic).

First, we determined the effects of rmPFC stimulation and lidocaine injection on reflexively evoked eyeblinks. Figure $5 A-C$ shows representative air-puff-evoked eyeblinks in controls after train stimulation of the rmPFC (Fig. 5B), and after a local lidocaine injection in the rmPFC (Fig. 5C). Quantitative analysis (Fig. 5) indicated that train stimulation of the rmPFC significantly increased the latency $(\mathrm{H}=12.946 ; p<0.001$; Fig. $5 D)$ and also significantly decreased the duration $\left(F_{(1,42)}=23.146 ; p \leq 0.001\right.$; Fig. $5 E)$, EMG area $\left(F_{(1,42)}=47.555 ; p \leq 0.001\right.$; Fig. $\left.5 F\right)$, and peak amplitude $(\mathrm{H}=9.137 ; p<0.001$; Fig. $5 G)$ of reflexively evoked blinks compared with values collected for control eyeblinks. In contrast, local lidocaine injection ( $5 \%$ at a rate of $0.1 \mu \mathrm{l} / \mathrm{min}$ ) in the rmPFC evoked eyeblinks with significantly shorter latency $(\mathrm{H}=$ 17.234; $p<0.001$; Fig. $5 D)$ and larger duration $\left(F_{(1,68)}=25.226 ; p \leq\right.$ 0.001; Fig. $5 E)$ and EMG area $\left[F_{(1,68)}=45.055 ; p \leq 0.001\right.$; Fig. $\left.5 F\right)$. However, EMG peak amplitude was not significantly modified $(\mathrm{H}=$ 2.753; $p=0.097$; Fig. $5 G$ ) compared with controls. Therefore, the activation of the rabbit rmPFC decreases reflexively evoked eyeblinks, whereas its inactivation increases them (Fig. 5).

Second, we checked the effects of rmPFC activation and inactivation on classically conditioned eyelid responses. As described 
in Materials and Methods, we implanted 12 additional rabbits with recording EMG electrodes in the orbicularis oculi muscle for the classical conditioning of eyelid responses. In addition, one group $(n=6)$ of these animals also had an stimulating electrode implanted in the contralateral rmPFC and the other group $(n=6$ animals) had an injection cannula implanted in the same selected rmPFC sites.

Half $(n=3)$ of the animals with implanted cannulas were injected with lidocaine (same amount and rate as described previously) during the second and third conditioning sessions, whereas the other half $(n=3)$ were injected with vehicle (Fig. $6 A, B, E)$. As shown in Figure $6 E$, lidocaine injection significantly $\left(F_{(9,27)}=14.049 ; p \leq 0.001\right)$ increased the acquisition of CRs and modified the kinematics of the evoked EMG responses of the orbicularis oculi muscle-i.e., there was a decrease in the latency of evoked CRs (Fig. 6G) and an increase in their duration (Fig. $6 H$ ) and total EMG area (Fig. 6I). Interestingly, and in opposition to results collected for reflex blinks, EMG peak amplitude was significantly $(t=2.258 ; p<0.05 ; \mathrm{H}=5.487 ; p=0.019)$ decreased (Fig. $6 J$ ), suggesting a disorganization of the conditioned EMG response-i.e., it was prevented from reaching large EMG peak values.

As shown in Figure 6, $C, D$, and $F$, half $(n=3)$ of the animals implanted with stimulating electrodes in the rmPFC contralateral to the conditioning side were stimulated during the sixth conditioning session, when asymptotic CR values had already been reached. It can be seen in Figure 6, $C$ and $D$, that train $(200 \mathrm{~Hz}, 50$ $\mathrm{ms}$ ) stimulation of the rmPFC $80 \mathrm{~ms}$ before US presentation prevented the appearance of a well defined CR and significantly $(t=2.966 ; p=0.08)$ decreased the expected percentage of CRs (Fig. $6 F$ ). Train stimulation of the rmPFC also modified the kinematic properties of EMG responses collected during the sixth conditioning session significantly compared with control values. Therefore, conditioned EMG responses evoked in stimulated animals were of significantly shorter duration $(t=11.992, p<$ 0.001 ; Fig. $6 H)$ and smaller peak amplitude $(t=12.097, p<$ $0.001 ; \mathrm{H}=48.649 ; p<0.001$; Fig. $6 \mathrm{~J})$. There were no significant changes in EMG latency $(t=0.00637 ; p=0.0995$; Fig. $6 G)$ or EMG area $(\mathrm{H}=0.138 ; p=0.71$; Fig. $6 I)$.

It is important to point out that the electrical stimulation of the rmPFC did not evoke any visually identifiable facial motor response apart from the reported inhibition of the EMG activity of the orbicularis oculi muscle. Therefore, the rmPFC must an inhibitory effect on the nearby anterior cingulate cortex (area 24c), which has been reported as having a primordial role in facial expression (Morecraft et al., 2001, 2007, 2012). Overall, it can be concluded that the rmPFC can modulate both the acquisition rate and the execution of classically conditioned eyelid responses, decreasing them when this cortical area is more active and increasing them when its activity is diminished.

\section{Discussion}

According to the present results, the rmPFC plays a preventing/ releasing role in the expression of acquired conditioned eyelid responses (Fig. 7), but it is not directly involved in the acquisition process or in the proper execution of CRs. Indeed, the rmPFC plays a depressant role when activated, evoking a freezing behavior in the rabbit (a typical prey animal), but as described previously (Leal-Campanario et al., 2007), this freezing of overt motor responses does not prevent the animal from acquiring eyelid CRs, only their motor expression. In contrast, the rmPFC plays an enhancing role in the expression of CRs when depressed, allowing its release, with a significantly shorter latency (Weiss and

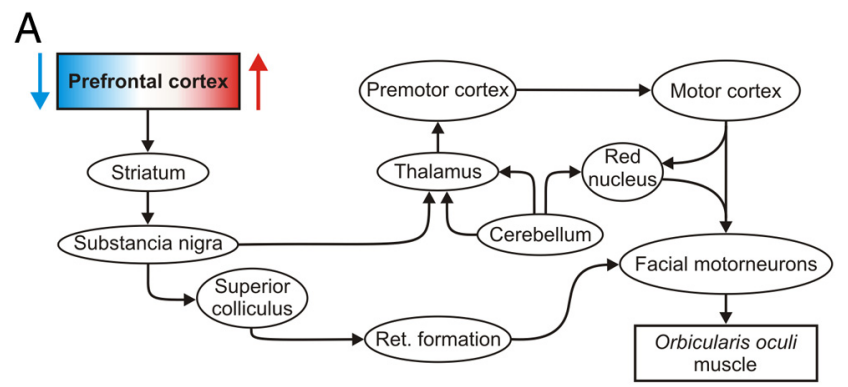

\section{B} PREFONTAL FIRING RATE

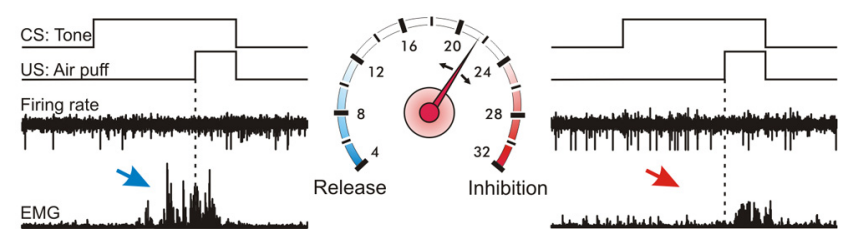

Figure 7. Schematic diagram of the putative functional role of rmPFC neurons. $\boldsymbol{A}$, Schematic diagram indicating the main pathways involved in the effects reported here of rmPFC activation or inactivation on reflex and conditioned eyelid responses. $\boldsymbol{B}$, Speedometer in the middle illustrates the firing rate of rmPFC neurons. An increase in neuronal firing rate (red part of the speedometer) inhibits the motor expression of the conditioned response (see red arrows), whereas a decrease (bluish part of the speedometer) allows the motor expression of the conditioned response (blue arrow). CS and US presentations are indicated, along with representative examples of neuronal firing rates and the rectified activities of the orbicularis oculi muscle.

Disterhoft, 2011). Therefore, the rmPFC seems not to be involved in the acquisition of the CR, but in the modulation of the level of expression of newly acquired motor abilities, including the emotional components of these learned responses. As described previously (Powell et al., 1996), the firing of medial PFC neurons is not directly related to the acquisition of pavlovian eyelid conditioning. The rmPFC of subprimates is a part of the orbital prefrontal cortex, considered to be the highest level of the limbic system and playing an important role in the proper activation or inhibition of selected behaviors and of attentive and cognitive processes (Fuster, 2001, 2008; Kolb et al., 2004).

\section{The permissive and/or modulatory role of the rmPFC on acquired eyelid CRs}

The present results suggest a differential role of the $\mathrm{rmPFC}$ in the acquisition of a classical eyeblink conditioning task compared with similar unitary recording and/or lesion studies performed in more caudal and/or more dorsolateral prefrontal areas (Weible et al., 2000, 2003, 2007; Weiss and Disterhoft, 2011). For example, neurons located in the caudal anterior cingulate cortex were shown to be involved early in the increased salience or relevance of CS presentations during trace eyeblink conditioning in the rabbit (Weible et al., 2003). In contrast, dorsolateral prefrontal cortex neurons present firing activities that persist during the CS-US interval in trace conditioning paradigms, helping in the generation of timed CRs (Weiss and Disterhoft, 2011). A specific population of cmPFC neurons recorded in rabbits also seems to bridge the temporal gap between the end of the CS and the beginning of the US during trace conditioning (Siegel et al., 2012). The persistent neural activity recorded in both dorsolateral and caudomedial PFC during the CS-US interval was probably generated by fast-spiking interneurons, and not projecting pyramidal prefrontal neurons (Povysheva et al., 2006). Other groups have reported that the lesion of the caudal part of the $\mathrm{mPFC}$ area confirmed its involvement in classical eyeblink conditioning 
when trace paradigms were used (Powell et al., 2005; Oswald et al., 2006).

The activity of the recorded rmPFC pyramidal neurons during the CS-US interval was not related significantly to the percentage of CRs and/or to the area of the rectified EMG activity of the orbicularis oculi muscle. In fact, their firing increased following CS presentations from the fourth to the sixth sessions and persisted to overlap with US presentation (Siegel et al., 2012). This significant increase in firing rate during CS-US presentations was in parallel with the increase in the percentage of CRs until reaching asymptotic values (Fig. 4C). Afterward, the firing rates of recorded rmPFC neurons returned to baseline (habituation sessions) values. Across training, the pyramidal cells recorded and included in this study could probably be activated by reentrant excitatory mediodorsal nucleus circuits carrying unspecific sensory information to increase attention to the ongoing paired CS-US presentations and to the timed release of the acquired motor responses. An interpretation of the putative role of rmPFC neurons in behaving rabbits is discussed below.

\section{A putative peculiar role of $\mathrm{rmPFC}$ in the rabbit}

The $\mathrm{mPFC}$ has been shown to be related to the affective components present in pavlovian conditioning (Powell et al., 1996) and in positive emotional learning (Burgdorf et al., 2011), and seems to have a specific role in the acquisition of the eyelid CRs using a trace paradigm depending upon selected US intensities (Oswald et al., 2006). Nevertheless, and from an ethological point of view, the rabbit is a typical prey species that presents a notable freezing behavior in the presence of their natural predators. Freezing is considered here as a cessation of all movements except those related to eye movements, breathing, and other autonomic activities (Misslin, 2003). This behavioral specialization could be dependent on a particular prefrontal function not present in other species of mammals (Elston, 2003; Jurado-Parras et al., 2012). Certainly, the electrical activation of the rmPFC reported here affected the percentage of CRs directly when presented during selected conditioning sessions. In contrast, the transient inactivation of the rmPFC produced an earlier presentation of shortlatency CRs. Interestingly, these significant changes in the expression of CRs seemed not to affect the acquisition process (Leal-Campanario et al., 2007), suggesting that the main role of the rmPFC in the rabbit could be in the control of the ongoing behavior depending on environmental and social constraints. The effects of rmPFC activation or inhibition could be mediated by prefrontal pyramidal cell projections to the amygdalar complex (Block et al., 1980; Quirk et al., 2003; Rosenkranz et al., 2003). It is known that electrical stimulation of the different amygdalar subnuclei can evoke selective fight-or-flight responses in cats (Block et al., 1980). The regulatory role of rmPFC neurons with regard to the amygdala can be further distributed into brainstem circuits involved in the expression of emotional behaviors by the wide descending projections of the central medial nucleus (Quirk et al., 2003; Rosenkranz et al., 2003; Likhtik et al., 2005).

\section{Connections of the rmPFC with neural centers involved in classical eyeblink conditioning}

The rmPFC of subprimates is homologous with the orbital area of the PFC of primates, both of them receiving a selective projection from the medial half of the thalamic mediodorsal nucleus (Benjamin et al., 1978; Kronforst-Collins and Disterhoft, 1998). According to Buchanan et al. (1994), the recording sites of rmPFC neurons reported here correspond to the rostral aspect of area 24 (anterior cingulate cortex) and to the rostrodorsal part of area 32 (prelimbic cortex), but apparently do not include the area $24 \mathrm{c}$ involved in facial expression in monkeys (Morecraft et al., 2001, $2007,2012)$. As indicated above, the recorded area of the rmPFC was identified by its heavy afferent projection originated in the medial half of the mediodorsal thalamic nucleus (Benjamin et al., 1978; Ray et al., 1992; Leal-Campanario et al., 2007). Recorded rmPFC neurons were antidromically and/or synaptically activated from this thalamic nucleus (Herry et al., 1999). It has been reported that short-latency synaptic activation of deep (V-VI) layers of the mPFC from the mediodorsal thalamic nucleus is produced by the activation of recurrent collaterals from prefrontal cortex/mediodorsal thalamic nucleus, whereas long-latency activation is probably the result of synaptic activation of superficial (II-III) cortical layers by mediodorsal thalamic neurons (Pirot et al., 1995).

The rmPFC is in a strategic position for controlling the generation and performance of selected motor responses. As described previously (Buchanan et al., 1994; Kronforst-Collins and Disterhoft, 1998; Fuster, 2001), prefrontal areas 24 and 32 project to the caudate nucleus and to the claustrum, from which prefrontal commands can reach many different sensorimotor cortical and subcortical areas. As indicated above, rmPFC areas also project to the mediodorsal thalamic nucleus and to other midline thalamic nuclei, regulating unspecific sensory inputs related to attentive processes and to the presence of novel sensory stimuli. In addition, PFC neurons project to different midbrain centers, including the substantia nigra pars reticulata, which are involved in movement initiation and coordination. Therefore, because of their firing properties and their selective connections, rmPFC neurons are suited to play a permissive and regulatory role in the generation of newly acquired motor responses.

From a functional point of view, the long-latency $(>80 \mathrm{~ms})$ inhibitory effects of rmPFC stimulation on both eyelid reflex and CRs suggest the involvement of polysynaptic pathways (Sauerland et al., 1967). For example, rmPFC neurons can modulate tectoreticular reflex blink circuits by their projections on basal ganglia and substantia nigra pars reticulata (Basso and Evinger, 1996; Basso et al., 1996). In addition, it seems there are prefrontal projections to the superior colliculus, which at its time project to both trigeminal and facial nuclei (Dauvergne et al., 2004). By their projection to pontine nuclei (Kronforst-Collins and Disterhoft, 1998; Siegel et al., 2012), PFC neurons can also regulate the participation of cerebellar circuits in the generation and/or performance of eyelid CRs. This projection is of particular interest because the cerebellum has long been implicated classically in the generation (Kim and Thompson, 1997) or performance (Jiménez-Díaz et al., 2004) of eyelid CRs induced with the help of delay paradigms.

In summary, these results provide new evidence about a sort of gating exerted by the rmPFC on the final expression of a basic reflex and its conditioned expression. This kind of control is very important to optimize goal-directed behaviors with no errors. Therefore, the rmPFC may exert a "flip-flop" mechanism to control the proper performance of the selected behavior against unwanted ones.

\section{References}

Alexander WH, Brown JW (2011) Medial prefrontal cortex as an actionoutcome predictor. Nat Neurosci 14:1338-1344. CrossRef Medline

Basso MA, Evinger C (1996) An explanation for reflex blink hyperexcitability in Parkinson's disease. II. Nucleus raphe magnus. J Neurosci 16:73187330. Medline

Basso MA, Powers AS, Evinger C (1996) An explanation for reflex blink 
hyperexcitability in Parkinson's disease. I. Superior colliculus. J Neurosci 16:7308-7317. Medline

Benjamin RM, Jackson JC, Golden GT (1978) Cortical projections of the thalamic mediodorsal nucleus in the rabbit. Brain Res 141:251-265. CrossRef Medline

Block CH, Siegel A, Edinger HM (1980) Effects of stimulation of the substantia innominata upon attack behavior elicited from the hypothalamus in the cat. Brain Res 197:57-74. CrossRef Medline

Buchanan SL, Thompson RH, Maxwell BL, Powell DA (1994) Efferent connections of the medial prefrontal cortex in the rabbit. Exp Brain Res 100:469-483. CrossRef Medline

Burgdorf J, Kroes RA, Weiss C, Oh MM, Disterhoft JF, Brudzynski SM, Panksepp J, Moskal JR (2011) Positive emotional learning is regulated in the medial prefrontal cortex by GluN2B-containing NMDA receptors. Neuroscience 192:515-523. CrossRef Medline

Clark GA, McCormick DA, Lavond DG, Thompson RF (1984) Effects of lesions of cerebellar nuclei on conditioned behavioral and hippocampal neuronal responses. Brain Res 291:125-136. CrossRef Medline

Corbit LH, Balleine BW (2003) The role of prelimbic cortex in instrumental conditioning. Behav Brain Res 146:145-157. CrossRef Medline

Dauvergne C, Ndiaye A, Buisseret-Delmas C, Buisseret P, Vanderwerf F, Pinganaud G (2004) Projections from the superior colliculus to the trigeminal system and facial nucleus in the rat. J Comp Neurol 478:233-247. CrossRef Medline

Dégenètais E, Thierry AM, Glowinski J, Gioanni Y (2002) Electrophysiological properties of pyramidal neurons in the rat prefrontal cortex: an in vivo intracellular recording study. Cereb Cortex 12:1-16. CrossRef Medline

Elston GN (2003) Cortex, cognition and the cell: new insights into the pyramidal neuron and prefrontal function. Cereb Cortex 13:1124-1138. CrossRef Medline

Fuster JM (2001) The prefrontal cortex-an update: time is of the essence. Neuron 30:319-333. CrossRef Medline

Fuster JM (2008) The prefrontal cortex, Ed 4. London: Academic.

Girgis M, Shih-Chang W (1981) A new stereotaxic atlas of the rabbit brain. St. Louis: Warren H. Green.

Gruart A, Schreurs BG, del Toro ED, Delgado-García JM (2000) Kinetic and frequency-domain properties of reflex and conditioned eyelid responses in the rabbit. J Neurophysiol 83:836-852. Medline

Herry C, Vouimba RM, Garcia R (1999) Plasticity in the mediodorsal thalamo-prefrontal cortical transmission in behaving mice. J Neurophysiol 82:2827-2832. Medline

Jiménez-Díaz L, Navarro-López J de D, Gruart A, Delgado-García JM (2004) Role of cerebellar interpositus nucleus in the genesis and control of reflex and conditioned eyelid responses. J Neurosci 24:9138-9145. CrossRef Medline

Jurado-Parras MT, Gruart A, Delgado-García JM (2012) Observational learning in mice can be prevented by medial prefrontal cortex stimulation and enhanced by nucleus accumbens stimulation. Learn Mem 19:99-106. CrossRef Medline

Kim JJ, Thompson RF (1997) Cerebellar circuits and synaptic mechanisms involved in classical eyeblink conditioning. Trends Neurosci 20:177-181. CrossRef Medline

Kolb B, Pellis S, Robinson TE (2004) Plasticity and functions of the orbital frontal cortex. Brain Cogn 55:104-115. CrossRef Medline

Kronforst-Collins MA, Disterhoft JF (1998) Lesions of the caudal area of rabbit medial prefrontal cortex impair trace eyeblink conditioning. Neurobiol Learn Mem 69:147-162. CrossRef Medline

Leal-Campanario R, Fairén A, Delgado-García JM, Gruart A (2007) Electrical stimulation of the rostral medial prefrontal cortex in rabbits inhibits the expression of conditioned eyelid responses but not their acquisition. Proc Natl Acad Sci U S A 104:11459-11464. CrossRef Medline

Likhtik E, Pelletier JG, Paz R, Paré D (2005) Prefrontal control of the amygdala. J Neurosci 25:7429-7437. CrossRef Medline

Mauk MD, Thompson RF (1987) Retention of classically conditioned eyelid responses following acute decerebration. Brain Res 403:89-95. CrossRef Medline

Misslin R (2003) The defense system of fear: behavior and neurocircuitry. Clin Neurophysiol 33:55-66. CrossRef Medline

Morecraft RJ, Louie JL, Herrick JL, Stilwell-Morecraft KS (2001) Cortical innervation of the facial nucleus in the non-human primate: a new inter- pretation of the effects of stroke and related subtotal brain trauma on the muscles of facial expression. Brain 124:176-208. CrossRef Medline

Morecraft RJ, McNeal DW, Stilwell-Morecraft KS, Gedney M, Ge J, Schroeder CM, van Hoesen GW (2007) Amygdala interconnections with the cingulate motor cortex in the rhesus monkey. J Comp Neurol 500:134165. CrossRef Medline

Morecraft RJ, Stilwell-Morecraft KS, Cipolloni PB, Ge J, McNeal DW, Pandya DN (2012) Cytoarchitecture and cortical connections of the anterior cingulate and adjacent somatomotor fields in the rhesus monkey. Brain Res Bull 87:457-497. CrossRef Medline

Múnera A, Gruart A, Muñoz MD, Fernández-Mas R, Delgado-García JM (2001) Hippocampal pyramidal cell activity encodes conditioned stimulus predictive value during classical conditioning in alert cats. J Neurophysiol 86:2571-2582. Medline

Nácher V, Ojeda S, Cadarso-Suárez C, Roca-Pardiñas J, Acuña C (2006) Neural correlates of memory retrieval in the prefrontal cortex. Eur J Neurosci 24:925-936. CrossRef Medline

Oswald B, Knuckley B, Mahan K, Sanders C, Powell DA (2006) Prefrontal control of trace versus delay eyeblink conditioning: role of the unconditioned stimulus in rabbits (Oryctolaguscuniculus). Behav Neurosci 120: 1033-1042. CrossRef Medline

Pirot J, Glowinski J, Thierry AM (1995) Excitatory responses evoked in prefrontal cortex by mediodorsal thalamic nucleus stimulation: influence of anesthesia. Eur J Neurosci 285:45-54. CrossRef Medline

Povysheva NV, Gonzalez-Burgos G, Zaitsev AV, Kröner S, Barrionuevo G, Lewis DA, Krimer LS (2006) Properties of excitatory synaptic responses in fast-spiking interneurons and pyramidal cells from monkey and rat prefrontal cortex. Cereb Cortex 16:541-552. CrossRef Medline

Powell DA, Maxwell B, Penney J (1996) Neuronal activity in the medial prefrontal cortex during pavlovian eyeblink and nictitating membrane conditioning. J Neurosci 16:6296-6306. Medline

Powell DA, Churchwell J, Burriss L (2005) Medial prefrontal lesions and pavlovian eyeblink and heart rate conditioning: effects of partial reinforcement on delay and trace conditioning in rabbits (Oryctolaguscuniculus). Behav Neurosci 119:180-189. CrossRef Medline

Quirk GJ, Likhtik E, Pelletier JG, Paré D (2003) Stimulation of medial prefrontal cortex decreases the responsiveness of central amygdala output neurons. J Neurosci 23:8800-8807. Medline

Ray JP, Russchen FT, Fuller TA, Price JL (1992) Sources of presumptive glutamatergic/aspartatergic afferents to the mediodorsal nucleus of the thalamus in the rat. J Comp Neurol 320:435-456. CrossRef Medline

Reiner A, Veenman CL, Medina L, Jiao Y, Del Mar N, Honig MG (2000) Pathway tracing using biotinylated dextran amines. J Neurosci Methods 103:23-37. CrossRef Medline

Romo R, Brody CD, Hernández A, Lemus L (1999) Neuronal correlates of parametric working memory in the prefrontal cortex. Nature 3:470-473. CrossRef Medline

Rosenkranz JA, Moore H, Grace AA (2003) The prefrontal cortex regulates lateral amygdala neuronal plasticity and responses to previously conditioned stimuli. J Neurosci 23:11054-11064. Medline

Sauerland EK, Knauss T, Nakamura Y, Clemente CD (1967) Inhibition of monosynaptic and polysynaptic reflexes and muscle tone by electrical stimulation of the cerebral cortex. Exp Neurol 17:159-171. CrossRef Medline

Shek JW, Wen GY, Wisniewski HM (1986) Atlas of the rabbit brain and spinal cord. Zurich: Karger.

Siegel JJ, Kalmbach B, Chitwood RA, Mauk MD (2012) Persistent activity in a cortical-to-subcortical circuit: bridging the temporal gap in trace eyelid conditioning. J Neurophysiol 107:50-64. CrossRef Medline

Simon B, Knuckley B, Churchwell J, Powell DA (2005) Post-training lesions of the medial prefrontal cortex interfere with subsequent performance of trace eyeblink conditioning. J Neurosci 25:10740-10746. CrossRef Medline

Takehara-Nishiuchi K, Kawahara S, Kirino Y (2005) NMDA receptordependent processes in the medial prefrontal cortex are important for acquisition and the early stage of consolidation during trace, but not delay eyeblink conditioning. Learn Mem 12:606-614. CrossRef Medline

Watanabe M (1990) Prefrontal unit activity during associative learning in the monkey. Exp Brain Res 80:296-309. Medline

Weible AP, McEchron MD, Disterhoft JF (2000) Cortical involvement in acquisition and extinction of trace eyeblink conditioning. Behav Neurosci 114:1056-1067. CrossRef Medline 
Weible AP, Weiss C, Disterhoft JF (2003) Activity profiles of single neurons in caudal anterior cingulate cortex during trace eyeblink conditioning in the rabbit. J Neurophysiol 90:599-612. CrossRef Medline

Weible AP, Weiss C, Disterhoft JF (2007) Connections of the caudal anterior cingulate cortex in rabbit: neural circuitry participating in the acqui- sition of trace eyeblink conditioning. Neuroscience 145:288-302. CrossRef Medline

Weiss C, Disterhoft JF (2011) Exploring prefrontal cortical memory mechanisms with eyeblink conditioning. Behav Neurosci 125:318-326. CrossRef Medline 